\title{
RADIO SOURCE COUNTS AT CENTIMETRE WAVELENGTHS
}

\author{
I. I. K. PAULINY-TOTH \\ Max-Planck-Institut für Radioastronomie, Bonn, F.R.G. \\ and \\ K. I. KELLERMANN \\ National Radio Astronomy Observatory, Green Bank, W.Va. 24944, U.S.A.*
}

\begin{abstract}
Counts of radio sources at $5 \mathrm{GHz}$ (6 cm wavelength) have been derived from a number of surveys including a new strong source survey. The source counts do not appear to differ markedly from an integral number-flux relation having a slope of -1.5 between 5 and $5 \times 10^{3}$ sources per steradian, and show a sharp drop at source densities smaller than $5 \mathrm{sr}^{-1}$. On the basis of the form of the number counts and the observed anisotropies in the distribution of sources and of their spectra, the cosmological significance of the source counts is questioned. In particular, the evidence for strong cosmic evolution appears weaker than is generally thought unless the cosmological origin of the quasar redshifts is assumed. Measurements of the radio spectra of the sources suggest a dependence of the spectral curvature on flux density.
\end{abstract}

\section{Introduction}

The present paper is concerned primarily with the results of a number of surveys carried out at a frequency of $5 \mathrm{GHz}$ at the National Radio Astronomy Observatory. The aim of these surveys has been to

(a) obtain the number-flux density relation at short wavelengths for comparison with those derived at metre wavelengths, and

(b) derive the number-flux density relation for different classes of sources, as defined by their spectral properties or optical identifications.

The surveys reported on previously are:

(i) a deep (D) survey made with the 300 -ft telescope complete above a flux density of $0.067 \mathrm{f}$.u. for an area of $3.77 \times 10^{-2} \mathrm{sr}$ and above $0.05 \mathrm{f}$.u. for an area of $3.63 \times 10^{-3} \mathrm{sr}$ (Davis, 1971, Paper I).

(ii) two strong source (S) surveys made with the 140-ft telescope and complete above 0.6 f.u.: the 'S2' survey covering 0.97 sr (Pauliny-Toth et al., 1972, Paper II) and the 'S3' survey, covering 1.14 sr (Pauliny-Toth and Kellermann, 1972, Paper III), as well as an earlier S1 survey, complete above $0.8 \mathrm{f}$.u. over an area of $0.27 \mathrm{sr}$ (Kellermann et al., 1968), and

(iii) an intermediate (I) survey made with the 140-ft telescope, covering $0.079 \mathrm{sr}$ down to a completeness limit of $0.25 \mathrm{f}$.u. (Paper II).

A discussion of the source counts based on these surveys has been published (Kellermann et al., 1971).

The present discussion includes data from a new strong source (S4) survey, made with the 300-ft telescope (Davis and Kellermann, 1974), which covers the northern sky between declinations $35^{\circ}$ and $70^{\circ}$, or a solid angle of $1.18 \mathrm{sr}$ for galactic latitudes

\footnotetext{
* Operated by Associated Universities, Inc., under contract with the National Science Foundation.
} 
$b \geqslant 20^{\circ}$. This new survey is believed to be complete down to at least the same level as the earlier $\mathbf{S}$ surveys; however, since only flux densities from the finding survey are at present available for the sources, the data are preliminary and larger corrections have had to be applied to the observed source counts for the effect of noise than for the $\mathbf{S 2}$ and $\mathbf{S 3}$ surveys. The area of sky covered by the $\mathbf{S}$ surveys to a completeness level of $0.6 \mathrm{f}$.u. is thus $3.29 \mathrm{sr}$.

In addition, accurate spectral indices at high frequencies have been obtained for the sources in the $\mathbf{S}$ and $\mathbf{D}$ surveys by measuring their flux densities at $2.7 \mathrm{GHz}$ at the National Radio Astronomy Observatory (Davis, 1974) and at 2.7 and $10.7 \mathrm{GHz}$ at the Max-Planck-Institut für Radioastronomie (Pauliny-Toth et al., 1974). Even for the sources from the $\mathbf{S 4}$ survey, the spectral information is accurate, since only the flux densities at 2.7 and $10.7 \mathrm{GHz}$ have been used to derive the spectral indices.

The results reported here are based on a total number of about 600 sources, so that their statistical accuracy is comparable to that of the Cambridge $408 \mathrm{MHz}$ data (Pooley and Ryle, 1968).

\section{The Source Counts}

The integral source counts at $5 \mathrm{GHz}$ show the familiar shape with a slope varying apparently smoothly from -1.5 at low flux densities to a steeper value at high flux densities. As shown in Table I, the best-fit slope for sources with a flux density greater than $0.6 \mathrm{f}$.u. (source density $124 \mathrm{sr}^{-1}$ ) is $-1.75 \pm 0.09$. Within the statistical uncertainties, the observed integral counts agree with those obtained from the $408 \mathrm{MHz}$ counts (Pooley and Ryle, 1968) and from the observed spectral index distribution of com-

\section{TABLE I}

Slope of the number-flux relation for the $\mathbf{S}$ surveys

Numbers in parentheses are the numbers of sources in the samples.

The spectral index in (b) is based on data at 2.7 and $10.7 \mathrm{GHz}$.

\begin{tabular}{|c|c|c|c|}
\hline Flux density & Whole survey & $b \geqslant 20^{\circ}$ & $b \leqslant-20^{\circ}$ \\
\hline \multicolumn{4}{|c|}{$\begin{array}{l}\text { (a) Flux density and } \\
\text { galactic latitude }\end{array}$} \\
\hline $\begin{array}{l}S \geqslant 0.6 \text { f.u. } \\
S \geqslant 1.0 \text { f.u. }\end{array}$ & $\begin{array}{l}-1.75 \pm 0.09(407) \\
-1.88 \pm 0.14(165)\end{array}$ & $\begin{array}{l}-1.90 \pm 0.12(246) \\
-1.89 \pm 0.20\end{array}$ & $\begin{array}{l}-1.55 \pm 0.12(161) \\
-1.86 \pm 0.21\end{array}$ \\
\hline
\end{tabular}

(b) Spectral index and galactic latitude

Spectral index

$\alpha \geqslant-0.5$

$-1.72 \pm 0.12(209)$

$-1.83 \pm 0.16(128)$

$-1.53 \pm 0.17(81)$

$\alpha<-0.5$

$-1.78 \pm 0.12(198)$

$-2.00 \pm 0.18(118)$

$-1.57 \pm 0.18(80)$

The slopes quoted in the table have been derived by the method of maximum likelihood (Crawford et al., 1970). 
plete samples of sources selected at $408 \mathrm{MHz}$, using the method described by Kellermann et al. (1968). Two conclusions can be drawn from this:

(i) that no major new class of radio sources appears at centimetre wavelengths, although the content of the surveys in terms of the optically associated objects and of the spectral properties of the sources is very different from that at meter wavelengths, and

(ii) that the $5 \mathrm{GHz}$ counts can be predicted on the basis of radio data alone, without reference to the red-shifts of the sources, to their evolution or to any particular cosmological model. Thus, any model which is chosen to reproduce the source counts and the spectral distribution at one wavelength will predict the source counts correctly at any other wavelength, so that the agreement of the observed and predicted counts at the latter wavelength does not provide independent confirmation of the model.

Although the integral counts are useful for such a comparison, they are misleading, both because of the statistical dependence of the data points and because any discontinuity in the counts in a particular range of flux densities is smeared out and propagated to much lower flux densities (e.g. Jauncey, 1967). The data from S, I and D surveys are shown in Figure 1 in the form of differential counts, normalized to a uniformly filled Euclidean model giving an integral source count $N_{0}=60 \mathrm{~S}^{-1.5} \mathrm{sr}^{-1}$

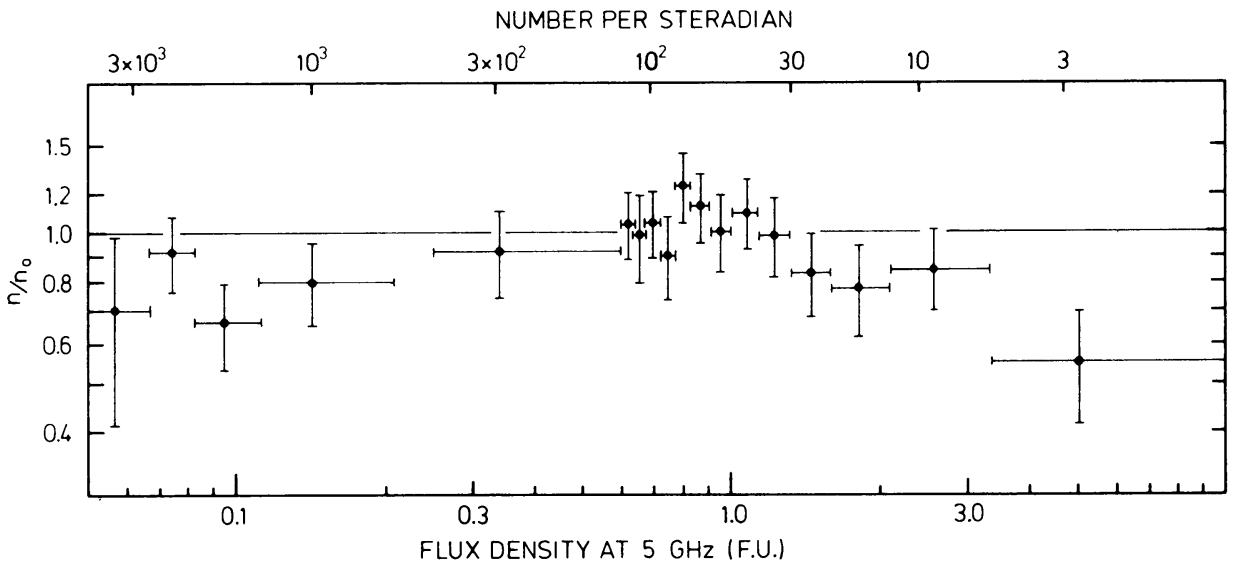

Fig. 1. Differential source counts from the $5 \mathrm{GHz} \mathbf{S}, \mathbf{I}$ and $\mathbf{D}$ surveys. The counts are normalised to those expected from a Euclidean model giving an integral count $N_{0}=60 S^{-1.5}$.

and the flux density intervals have been chosen so that this model should give approximately equal numbers of sources in each interval.

It is clear from Figure 1 that the statistical fluctuations in the counts are large. The counts do not differ significantly from those expected on the basis of a static Euclidean model between source densities of $5 \times 10^{3}$ and $30 \mathrm{sr}^{-1}$ and the difference is statistically significant only below $5 \mathrm{sr}^{-1}$. The steep slope of the integral counts for the $\mathbf{S}$ surveys as a whole (Table I), the departure of which from the Euclidean 
value is formally statistically significant at a level of 2 to $3 \sigma$, in fact depends critically on the relatively low number density of the strongest sources $(S \geqslant 3.3 \mathrm{f}$.u.). The addition of only 6 sources $\mathrm{sr}^{-1}$ in this flux density range, or about 70 over the whole sky, is sufficient to reproduce the Euclidean slope over the whole range of flux densities observed. It is important to note the sharpness of the decrease in the differential counts for the strong sources, which is also observed at other wavelengths (Kellermann, 1972) but which is masked when only the integral counts are considered. In view of the wide range in the radio luminosities of even the radio galaxies, it appears difficult to explain this sharp decrease as any cosmological effect.

The form of the differential counts, at least in this range of source densities, does not present compelling evidence for any strong evolutionary effects, but rather appears remarkably consistent with a static Euclidean universe with a relatively small number of missing strong sources. It has been argued that this deficiency cannot be treated as local and that even a slope of -1.5 is evidence for evolution. Both these arguments, however, assume the cosmological interpretation of the redshifts of the quasars or the more exotic (e.g. $N$-type) galaxies or that the unidentified sources are distant bright galaxies rather than nearby subluminous objects. If these redshifts are not of cosmological origin so that most radio sources are at distances comparable to those of the other identified radio galaxies, then there is no need to invoke evolution to explain the observed source counts in the range of source densities considered.

Some support for the latter interpretation is given by the source counts for the identified quasars and galaxies in the $\mathbf{S}$ surveys, the slope for both classes of sources being close to -1.5 . The steep slope for all the sources in the $\mathbf{S}$ surveys is due to the unidentified sources, the spectral properties of which suggest that they may be a mixture of quasars and radio galaxies in roughly equal numbers (Paper III). If this is so, then the slope for both classes of objects is close to -1.8 , so that the deficiency in the number of strong sources is due equally to quasars and radio galaxies and the two classes of sources appear to sample the same volume of space, a result which seems inconsistent with the large apparent difference in their mean redshifts.

\section{The Spectral Index Distribution}

As has been shown in Paper III, the distribution of the spectral indices for a complete sample of sources at $5 \mathrm{GHz}$ is a function of the limiting flux density of the sample. The median spectral index * derived from data at $5 \mathrm{GHz}$ and $408 \mathrm{MHz}$ changes from $-0.30 \pm 0.06$ for a sample with $S_{5 \mathrm{GHz}} \geqslant 1.5$ f.u. to $-0.63 \pm 0.04$ for a sample with $0.067 \leqslant S_{5 \mathrm{GHz}}<0.1 \mathrm{f}$.u., the fraction of sources with $\alpha \geqslant-0.5$ changing from 57 to $40 \%$ respectively. It was also shown that this dependence of the median spectral index on flux density can be obtained from the number counts at $408 \mathrm{MHz}$ and the distribution of the spectral index $\alpha(408 \mathrm{MHz}-5 \mathrm{GHz})$ for sources selected at $408 \mathrm{MHz}$.

* The spectral index, $\alpha$, is defined by $S x v^{x}$ where $v$ is the frequency. 
It must again be emphasised that this prediction of the spectral index distribution for sources selected at $5 \mathrm{GHz}$ is based on radio data alone, independently of any model of the Universe or of the evolution of the sources, so that a correct prediction of this distribution by a particular model does not provide independent evidence for the correctness of the model.

The accurate high-frequency spectral indices obtained from the measurements at 10.7 and $2.7 \mathrm{GHz}$ have been used to investigate the behaviour of the spectral index distribution further. The distributions for all the sources in the $\mathbf{S}$ surveys based on these measurements are shown in Figure 2. Both distributions clearly show two dis-

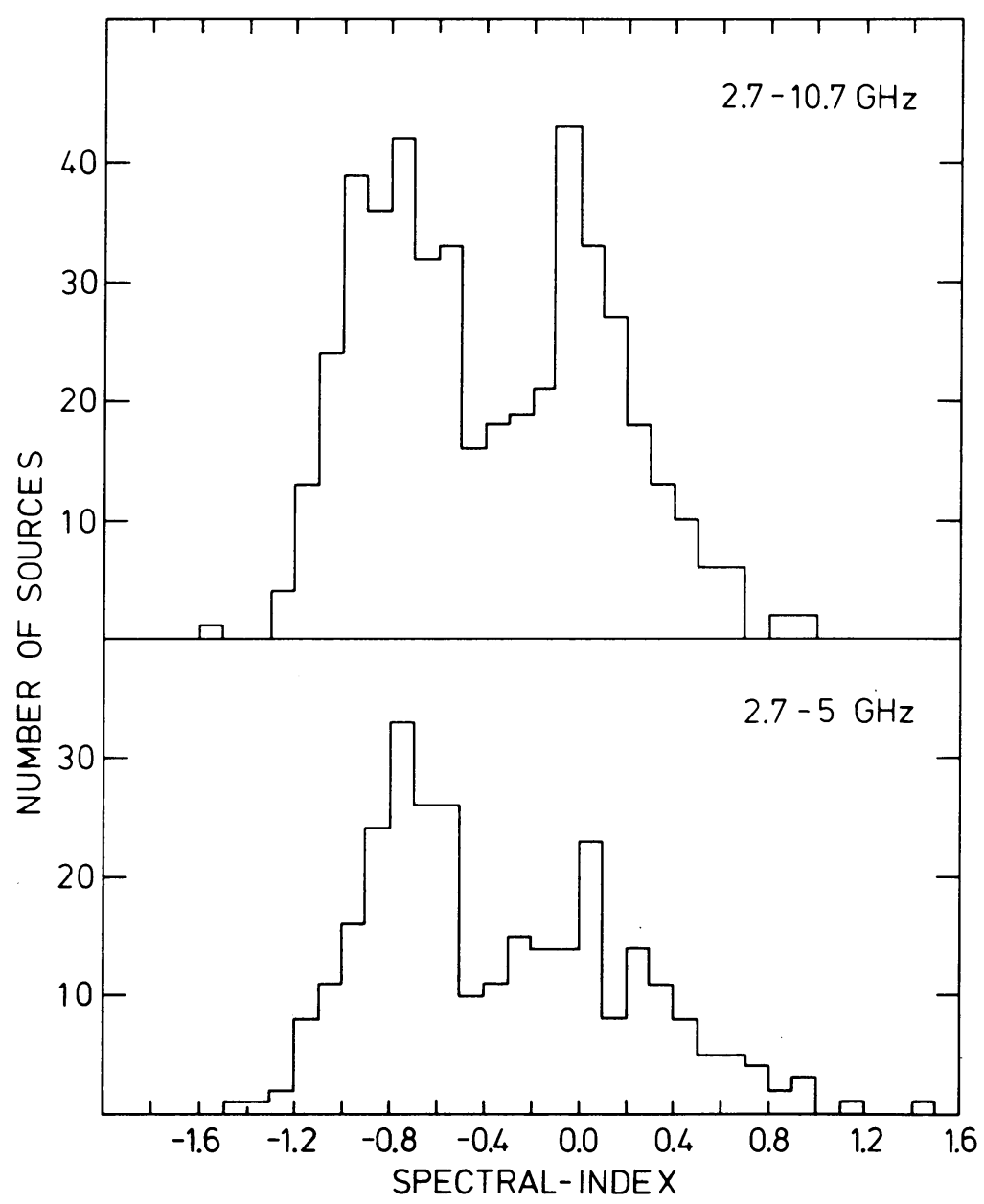

Fig. 2. The spectral index distributions for the $5 \mathrm{GHz} \mathbf{S}$ surveys. The top part shows the distribution for the S1, S2, S3 and S4 surveys based on flux densities at 2.7 and $10.7 \mathrm{GHz}$; the bottom part for the $\mathbf{S 1}, \mathbf{S 2}$ and S3 surveys based on the $5 \mathrm{GHz}$ and $2.7 \mathrm{GHz}$ data. Both distributions are for sources having flux densities above 0.6 f.u. at $5 \mathrm{GHz}$. 
tinct peaks near $\alpha=-0.8$ and $\alpha=0$, so that a separation of the sources into classes with $\alpha \geqslant-0.5$ and $\alpha<-0.5$ appears to be physically meaningful. If this separation is made, it is found that the slope of the source counts for the two classes is very nearly the same (Table I). Consistent with this, no dependence of the median spectral index, or of the fraction of sources having flat spectra on the flux density at $5 \mathrm{GHz}$ is found (Table II).

\section{TABLE II}

Spectral index distribution as a function of the flux density $S$ at $5 \mathrm{GHz}$; (a) for the $\mathbf{S 1}, \mathbf{S 2}, \mathbf{S 3}$ and $\mathbf{S 4}$ surveys using data at 2.7 and $10.7 \mathrm{GHz}$; (b) for the $\mathbf{S 1}, \mathbf{S 2}, \mathbf{S 3}$ and $\mathbf{D}$ surveys using data at 2.7 and $5 \mathrm{GHz}$ and (c) for comparison, the distribution for S1, S2, S3 and D surveys using data at $408 \mathrm{MHz}$ and $5 \mathrm{GHz}$ (Paper III)

\begin{tabular}{|c|c|c|c|c|c|}
\hline & $\begin{array}{l}\text { Flux density } \\
\text { range }\end{array}$ & Median index & Dispersion & $\begin{array}{l}\text { Fraction of } \\
\text { sources with } \\
\alpha \geqslant-0.5\end{array}$ & $\begin{array}{l}\text { Number of } \\
\text { sources }\end{array}$ \\
\hline \multirow[t]{4}{*}{ (a) } & $S \geqslant 1.5$ & $-0.47 \pm 0.06$ & 0.50 & 51 & 84 \\
\hline & $1.0 \leqslant S<1.5$ & $-0.34 \pm 0.05$ & 0.53 & 57 & 106 \\
\hline & $0.8 \leqslant S<1.0$ & $-0.52 \pm 0.05$ & 0.54 & 49 & 98 \\
\hline & $0.6 \leqslant S<0.8$ & $-0.51 \pm 0.04$ & 0.50 & 50 & 168 \\
\hline \multirow[t]{6}{*}{ (b) } & $S \geqslant 1.5$ & $-0.53 \pm 0.07$ & 0.50 & 47 & 58 \\
\hline & $1.0 \leqslant S<1.5$ & $-0.35 \pm 0.07$ & 0.57 & 52 & 67 \\
\hline & $0.8 \leqslant S<1.0$ & $-0.48 \pm 0.07$ & 0.53 & 51 & 63 \\
\hline & $0.6 \leqslant S<0.8$ & $-0.48 \pm 0.06$ & 0.58 & 51 & 106 \\
\hline & $0.1 \leqslant S<0.6$ & $-0.46 \pm 0.06$ & 0.53 & 52 & 75 \\
\hline & $0.067 \leqslant S<0.1$ & $-0.43 \pm 0.05$ & 0.43 & 42 & 69 \\
\hline \multirow[t]{6}{*}{ (c) } & $S \geqslant 1.5$ & $-0.30 \pm 0.06$ & 0.48 & 57 & 58 \\
\hline & $1.0 \leqslant S<1.5$ & $-0.35 \pm 0.05$ & 0.40 & 60 & 68 \\
\hline & $0.8 \leqslant S<1.0$ & $-0.54 \pm 0.06$ & 0.46 & 47 & 64 \\
\hline & $0.6 \leqslant S<0.8$ & $-0.55 \pm 0.05$ & 0.47 & 45 & 108 \\
\hline & $0.1 \leqslant S<0.6$ & $-0.60 \pm 0.07$ & 0.46 & 43 & 46 \\
\hline & $0.067 \leqslant S<0.1$ & $-0.63 \pm 0.04$ & 0.31 & 40 & 53 \\
\hline
\end{tabular}

Thus an $\alpha-S$ dependence is found when the spectral index $\alpha$ is based on data at $5 \mathrm{GHz}$ and at $408 \mathrm{MHz}$, but not when it is based on measurements near $5 \mathrm{GHz}$. The effect is not due to systematic errors, which would have to amount to $50 \%$ at 2.7 or $10.7 \mathrm{GHz}$ and $100 \%$ at $408 \mathrm{MHz}$. The observations therefore imply a systematic change in the mean spectral curvature with the flux density. The change is in the sense that the spectra for the stronger sources $\left(S_{5 \mathrm{GHz}} \geqslant 1\right.$ f.u.) tend to become flatter at low frequencies, while those of the weaker sources $\left(S_{5 \mathrm{GHz}}<0.6 \mathrm{f}\right.$.u. $)$ tend to become steeper.

\section{Isotropy}

With the addition of the $\mathbf{S} \mathbf{4}$ survey, the area of sky covered has been extended, mainly 
in the north galactic hemisphere. In order to check the previously reported evidence for differences in the source counts in the north and south galactic hemisphere (Paper III; Yahil, 1972) the data have been reexamined and the results for the $\mathbf{S}$ surveys are summarised in Table I.

For the sources having $S_{5 \mathrm{GH}} \geqslant 0.6$ f.u., the difference between the slopes of the source counts in the two hemispheres is $0.35 \pm 0.17$, the slope in the south hemisphere being close to the Euclidean value. The total source density down to 0.6 f.u. is the same for the two hemispheres within the statistical errors, so that the difference in the slopes implies a relative deficiency of strong sources in the north galactic hemisphere. This is confirmed if the differential counts are examined separately for the two hemispheres. The distribution of the strongest sources thus shows an anomaly on an angular scale of $2 \pi \mathrm{sr}$, the magnitude of the anomaly being of the same order as the departure of the total source counts from those expected on the basis of the Euclidean model.

When the source counts are examined separately for the two classes of sources having $\alpha(2.7 \mathrm{GHz}-10.7 \mathrm{GHz})$ greater or less than -0.5 , the slopes for the two classes do not differ significantly within either hemisphere (Table I). As shown in Paper III, however, when the division is made on the basis of $\alpha(5 \mathrm{GHz}-408 \mathrm{MHz})$, the steep slope in the north galactic hemisphere is due largely to a relative deficiency of strong sources with steep spectra in this frequency range. The two results together imply that the dependence of spectral curvature on flux density is confined to the northern hemisphere. Thus anomalies in the spectral properties of radio sources are also present on an angular scale of $2 \pi \mathrm{sr}$.

Evidence for further anisotropy for weaker sources and on smaller angular scales has been given by Davis (1971) and Maslowski et al. (1973). It is clearly difficult to reconcile the existence of such anisotropies in the distribution and properties of radio sources with the cosmological significance of the source counts.

\section{Summary}

Comparison of the differential source counts from surveys at metre and centimetre wavelengths (e.g. Kellermann, 1972; Longair, 1974) shows that they are essentially in agreement, but that the steep slope for the strongest sources may be less marked at centimetre wavelengths. This difference can be attributed to the fact that most of the sources in the metre-wavelength surveys have steep spectra and it is for these sources that the deficiency of strong sources is most pronounced. The anisotropy in the slope of the number counts for the strong sources is, however, apparently not present at metre wavelengths (Longair, 1974).

The particular question raised by the radio observations at short wavelengths, for which any cosmological theory must provide an adequate explanation, are:

(a) the Euclidean slope of the source counts over a large range of source densities;

(b) the sharp discountinuity in the differential counts near $5 \mathrm{sr}^{-1}$;

(c) the similarity of the slopes found for the counts of radio galaxies and quasars; 
(d) the complex behaviour of the spectral distribution as a function of frequency and flux density, and

(e) the anisotropies in the angular distribution and spectra of the sources.

It seems to us that the observations are consistent with a non-evolving model and that the apparent steep slope for the strongest sources may be due to a relatively small deficiency of sources. Since this deficiency does not appear to be isotropic, it can be interpreted as local without giving the Earth a privileged position in the Universe. Such a model is naturally inconsistent with the cosmological origin of the redshifts of quasars and hence with the cosmological relevance of the source counts themselves.

\section{References}

Crawford, D. E., Jauncey, D. L., and Murdoch, H. S.: 1970, Astrophys. J. 162, 405.

Davis, M. M.: 1971, Astron. J. 76, 980.

Davis, M. M.: 1974, (in preparation).

Davis, M. M. and Kellermann, K. I.: 1974 (in preparation).

Jauncey, D. L. : 1967, Nature 217, 877.

Kellermann, K. I.: 1972, Astron. J. 77, 531.

Kellermann, K. I., Davis, M. M., and Pauliny-Toth, I. I. K.: 1971, Astrophys. J. Letters $170, \mathrm{L1}$.

Kellermann, K. I., Pauliny-Toth, I. I. K., and Davis, M. M.: 1968, Astrophys. Letters 2, 113.

Longair, M. S. : 1974, this volume, p. 93.

Maslowski, J.: 1972, Astron. Astrophys. 16, 197.

Maslowski, J., Machalski, J., and Zieba, S.: 1973, Astron. Astrophys. 28, 289.

Pauliny-Toth, I. I. K. and Kellermann, K. I. : 1972, Astron. J. 77, 560.

Pauliny-Toth, I. I. K., Preuß, E., Witzel, A., and Kellermann, K. I. : 1974, (in preparation).

Pauliny-Toth, I. I. K., Kellermann, K. I., Davis, M. M., Fomalont, E., and Shaffer, D. : 1972, Astron. J. 77, 265.

Pooley, G. G. and Ryle, M.: 1968, Monthly Notices Roy. Astron. Soc. 139, 515.

Yahil, A.: 1972, Astrophys. J. 178, 45.

\section{DISCUSSION}

Schmidt: If the quasars are local, then their $N(S)$ will have a slope of 1.5 and then the radio galaxies would be solely responsible for the observed slope of 1.8. Hence there would be even more need for radio galaxy evolution in this case.

Pauliny-Toth: If the unidentified sources are divided equally among the quasars and radio galaxies, the slope is about -1.8 for each class. This steep slope is, however, largely due to the deficiency of strong sources, in each case.

Rees: Dr Longair emphasized the importance of considering the radio data as a whole rather than concentrating on a particular frequency. Do you think that your interpretation in terms of a 'local hole' can also be reconciled with the $408 \mathrm{MHz}$ counts?

Pauliny-Toth: If the differential counts for the available frequencies are plotted together, as was done by Kellermann (1972, reference in paper) there is no significant difference between them, considering the statistical uncertainties.

Longair: What do you mean by the Euclidean slope of the source counts over a large range of flux densities in view of the evidence of the $5 \mathrm{C}$ and Westerbork surveys which show that the counts converge dramatically at small flux densities?

Pauliny-Toth: I mean that the normalised differential counts are flat between source densities of $5 \times 10^{3}$ and $5 \mathrm{sr}^{-1}$, the range from which the evidence for evolution was originally suggested.

Kiang: What is the size of this Local Hole? And how does it compare with the size of superclusters we were talking about?

Pauliny-Toth: From the $5 \mathrm{GHz}$ counts, nothing can be said at present, because of the high proportion 
of unidentified sources even among the strong sources. Assigning a size of course means interpreting the redshifts as being of cosmological origin.

Longair: The size of the 'local hole' can be estimated from the source counts at $408 \mathrm{MHz}$ without making any assumption about the nature of the redshifts of the objects counted. The slope of the source counts in the region of convergence gives an estimate of the redshift of these objects if it is assumed that one is dealing with a single luminosity class. One can then work out the distance of the sources at the 'edge' of the local hole from the lowest flux density at which the deficit of sources persists. This gives a minimum size for the hole of $z=0.03$. If one assumes there is dispersion in the intrinsic luminosity of radio sources (as we know there is) the size of the hole has to be increased, or the hole will be washed out. Conservatively, the hole must extend to $z=0.3$ so that the hole must be a cosmological effect on the largest scale and mimic closely an evolutionary model.

Schmidt: The 'deficiency' of bright radio sources leads to an artificial picture of the source distribution because the luminosity function of radio galaxies is very wide. The local low-density region will have a size that increases strongly with radio luminosity.

Arp: The absolute isotropy school misses some important clues, I believe. In addition to the radio evidence you have presented, there is optical evidence. If you look at the apparent magnitudes of the QRSs in the north galactic hemisphere you find they are on the average one magnitude brighter than in the south galactic hemisphere. Of course, there is one other well known optical anisotropy - that of bright galaxies $\left(m_{p g}<12.0\right)$ which are much denser in the northern galactic than the southern galactic hemisphere. 